\title{
On stability analysis of nonlinear discrete singularly perturbed T-S fuzzy models
}

\author{
Boutheina Sfaihi · Mohamed Benrejeb
}

Received: 19 November 2012 / Revised: 14 February 2013 / Accepted: 15 February 2013 / Published online: 14 March 2013

(C) Springer-Verlag Berlin Heidelberg 2013

\begin{abstract}
This paper investigates the stability problem of a class of discrete-time singularly perturbed Tagagi-Sugeno (T-S) fuzzy models. Stability conditions of reduced slow models, based on the use of Borne and Gentina practical stability criterion and matrices in the arrow form, are developed and compared with those concerning the initial singularly perturbed T-S system. The obtained results are practical and easy to use. An example is introduced to illustrate the proposed approaches.
\end{abstract}

Keywords Discrete T-S fuzzy model .

Singularly perturbed systems · Order reduction · Stability · Arrow form matrix · Vector norms

\section{Introduction}

Some small physical parameters such as time constants, masses, capacitances, etc, increase the order of dynamic systems and introduce the multi-time scales property. Resulting systems can possess simultaneously, slow and fast coupling states increasing the system complexity. The singular perturbation approach [1-4] is a powerful technique for systems order reduction and time scales separation. The method explicit the time scale separation by mean of a small singular perturbation parameter $\mu$. When $\mu$ is small enough, the high order system is decomposed into slow and fast subsystems and considered as a singularly perturbed system.

B. Sfaihi $(\varangle) \cdot$ M. Benrejeb

Laboratoire de Recherche en Automatique (LARA), Université

de Tunis El Manar, Ecole Nationale d'Ingénieurs de Tunis,

B.P. 37, 1002 Tunis, Le Belvédère, Tunisia

e-mail: boutheina.sfaihi@isetr.rnu.tn

M. Benrejeb

e-mail: mohamed.benrejeb@enit.rnu.tn
Stability of linear singularly perturbed systems have been extensively studied in past years and a great number of results have been reported in the literature; see, e. g. [5-11], and the references therein. Recently, a great amount of effort has focused on the stability analysis of nonlinear singularly perturbed systems [12-23] where the properties of two lower order slow and fast subsystems are studied by mean of Lyapunov functions to predict the stability properties of the composite system.

In spite of the progression of stability nonlinear singularly perturbed systems analysis, it is not that obvious to apply complex techniques to practical engineering problems. It is still needed to develop the more simple stability technique for general nonlinear singularly perturbed systems. The fuzzy control theory $[24,25]$ uses collections of linguistic rules in order to model such as systems by considering qualitative aspects of human knowledge and reasoning processes without employing a precise quantitative analysis [26].

Fuzzy set theory has been developed and widely studied in the past two decades. It has been successfully applied in engineering problems due to its capacity of modeling and controlling complex nonlinear systems [27,28]. The most known methods in the literature designed for synthesizing stability conditions of fuzzy systems are [29-31]: Popov's stability criterion [32,33], the circle criterion [34,35], conicity criterion (extended version of circle criterion) [36,37], direct Lyapunov's method [38-40], analysis of system stability in phase space [35,41], the describing function method [37], methods of stability indices and systems robustness [37,35], methods based on theory of input-output stability [37,42], hyperstability theory [43-45] and heuristic methods [37,46].

Recently, stability of singular fuzzy systems have been investigated [47-55]. Huang [47] proposes a discrete singular T-S (DST-S) model and introduces to stability criteria by non-strict linear matrix inequalities (LMIs) and projection 
method. Liu et al. [48] synthesizes stability conditions of DST-S systems in term of LMIs and derives stability conditions for feedback controller via the nonlinear matrix inequalities (NMIs). Dong and Yang [49] present a method of evaluating the upper bound of the singular perturbation parameter $\mu$ for DST-S systems with meeting a prescribed $\mathrm{H} \infty$ performance bound requirement. Xu and Lam [50] propose a necessary and sufficient stability condition for uncertain DST-S systems in terms of a strict linear matrix inequality. $\mathrm{Xu}$ et al. [51] considers the problem of robust stability of uncertain DST-S systems with time-varying norm-bounded parameter uncertainties. A sufficient stability condition is proposed in terms of a set of LMIs. Chen et al. [52] treat state feedback robust stabilization problems for DST-S systems with parameter uncertainty, based on a matrix spectral norm approach.

Motivated by the fact that fuzzy sets provide an effective way to describe a nonlinear system, we will investigate, in this paper, the stability problem for T-S fuzzy discrete singular perturbed systems without using conventional Lyapunov function. New sufficient stability conditions, for original and reduced order discrete nonlinear T-S fuzzy models, are developed based on the arrow matrix form and Borne and Gentina criterion.

This paper is organized as follows. In sect. 2, the fuzzy system modeling and decoupling procedure are formulated via the singular perturbation technique. Section 3, stability conditions based on Lyapunov functions are reviewed, and new stability conditions for T-S fuzzy discrete singularly perturbed systems are proposed. In sect. 4, a numerical example is given, and finally, conclusions are presented in sect. 5 .

\section{Two-time scale singularly perturbed fuzzy model description}

Physical processes are very complex in practice and rigorous mathematical models can be very difficult to synthesize, if not impossible. Many of these systems can be expressed in some form of mathematical model locally, or as an aggregation of a set of mathematical models. Here, we consider the Takagi-Sugeno (T-S) model to represent a complex system that includes local analytic nonlinear models $S_{i}$ [56]. The $i$ th fuzzy inference rule of the fuzzy model is of the following form:

$$
\begin{aligned}
& R_{i}: I F x_{k} \text { is } M_{1}^{i} \cdots \text { and } x_{k} \text { is } M_{n}^{i} \text { THEN } \\
& x_{k+1}=A_{i} \text { (.) } x_{k}, \quad i \in I:=1,2, \cdots, m
\end{aligned}
$$

where the state vector $x(k T)$ is noted $x_{k}, x_{k} \in \mathbb{R}^{n}, k T$ is the discrete time and $T$ the sampling time such that $x_{k}=$ $\left[\begin{array}{ll}x_{k}^{1^{T}} & x_{k}^{2^{T}}\end{array}\right]^{T} \cdot x_{k}^{1} \in \mathbb{R}^{n_{1}}, x_{k}^{2} \in \mathbb{R}^{n_{2}}$ and $m$ denotes the number of inference rules and $M_{j}^{i}(j=1,2, \ldots, n)$ the fuzzy sets.
The instantaneous characteristic $n \times n$ matrix $A_{i}$ (.) of the $i$ th local model of the studied system is defined by

$A_{i}()=.\left[\begin{array}{cc}A_{i, 11}(\cdot) & A_{i, 12}(\cdot) \\ A_{i, 21}(\cdot) & A_{i, 22}(\cdot)\end{array}\right]$

By using a standard fuzzy inference method -that is, using a singleton fuzzifier, product fuzzy inference and weighted average defuzzifier- the final state of the fuzzy system $S$ is inferred as follows [31]

$S: x_{k+1}=\sum_{i=1}^{m} h_{i}\left(x_{k}\right) A_{i}(\cdot) x_{k}$

with

$h_{i}\left(x_{k}\right)=\frac{w_{i}\left(x_{k}\right)}{\sum_{i=1}^{m} w_{i}\left(x_{k}\right)}$ and $w_{i}\left(x_{k}\right)=\prod_{j=1}^{n} M_{j}^{i}$

We assume that $w_{i}\left(x_{k}\right) \geqslant 0$ and $\sum_{i=1}^{m} w_{i}\left(x_{k}\right)>0$ for $i \in I$. Then, it is easy to see that $h_{i}\left(x_{k}\right) \geqslant 0$, for $i \in I$ and $\sum_{i=1}^{m} h_{i}\left(x_{k}\right)=1$.

The local system $S_{i}$ is assumed to possess a two-timescale property, which means that the $n$ eigenvalues of $S_{i}$ can be separated into $n_{1}$ slow modes and $n_{2}$ stable fast modes related to $x_{k}^{1}$ and $x_{k}^{2}$, respectively. The fast subsystem $x_{k}^{2}$, assumed to be stable, is active only during a short initial period, after, it is negligible and the system can be described by it slow subsystem $x_{k}^{1}$ [57].

Often, numerical methods for simulation or controller design cannot be applied to large scale systems because of their extensive numerical costs. This motivates model reduction, which is the approximation of the original, large realization by a realization of smaller order. A method that maintains the coordinate system of the original model is based on singular perturbation technique $[1,5,6]$. In most classical and modern control schemes, singular perturbation techniques exploit the two-time-scale nature of the system in order to decompose the design problem into slow and fast modes.

Singularly perturbed systems have the following form [6, 8,58-60]

$\left[\begin{array}{c}x_{k+1}^{1} \\ x_{k+1}^{2}\end{array}\right]=\left[\begin{array}{cc}I_{n 1}+\mu A_{i, 11}^{*} & \mu A_{i, 12}^{*} \\ A_{i, 21}^{*} & A_{i, 22}^{*}\end{array}\right]\left[\begin{array}{c}x_{k}^{1} \\ x_{k}^{2}\end{array}\right]$

where $\mu$ is a small positive singular perturbation parameter that indicates separation of the state space variables into slow variables $x_{k}^{1}$ and fast variables $x_{k}^{2}$, and det $\left(I_{n_{2}}-A_{i, 22}^{*}\right) \neq 0$ [1]. The slow subsystem is defined by neglecting the fast stable dynamics, which is equivalent to replace the second equation of (5) by its steady-state algebraic equation. The fast subsystem, supposed to be stable, is derived by assuming that slow variables are constant during fast transients and $\mu=0$. 
Described system (5) is dual to system (1) and it is possible to put the system into the singularly perturbed form (5). The relation ship among the system matrices defined in (1) and in (5) are as follows

$$
\begin{aligned}
& A_{i, 11}^{*}=\mu^{-1}\left(A_{i, 11}-I_{n_{1}}\right), A_{i, 12}^{*}=\mu^{-1} A_{i, 12} \\
& A_{i, 21}^{*}=A_{i, 21}, \quad A_{i, 22}^{*}=A_{i, 22}
\end{aligned}
$$

Applying the decoupling transformation $[1,6,61,62]$ defined by

$$
\begin{aligned}
& {\left[\begin{array}{c}
x_{k+1}^{s} \\
x_{k+1}^{f}
\end{array}\right]=\left[\begin{array}{cc}
I_{n_{1}}-\mu M_{i} L_{i} & -\mu M_{i} \\
L_{i} & I_{n_{2}}
\end{array}\right]\left[\begin{array}{l}
x_{k+1}^{1} \\
x_{k+1}^{2}
\end{array}\right]} \\
& {\left[\begin{array}{l}
x_{k+1}^{1} \\
x_{k+1}^{2}
\end{array}\right]=\left[\begin{array}{cc}
I_{n_{1}} & \mu M_{i} \\
-L_{i} & I_{n_{2}}-\mu L_{i} M_{i}
\end{array}\right]\left[\begin{array}{c}
x_{k+1}^{s} \\
x_{k+1}^{f}
\end{array}\right]}
\end{aligned}
$$

the singularly perturbed system (5) can be decoupled into independent slow and fast subsystems [6] as

$S_{i}^{d}:\left[\begin{array}{c}x_{k+1}^{s} \\ x_{k+1}^{f}\end{array}\right]=\left[\begin{array}{cc}I_{n_{1}}+\mu A_{i}^{s} & 0 \\ 0 & A_{i, 22}^{*}\end{array}\right]\left[\begin{array}{l}x_{k}^{s} \\ x_{k}^{f}\end{array}\right]$

$S_{i}^{s}: x_{k+1}^{s}=\left(I_{n_{1}}+\mu A_{i}^{s}\right) x_{k}^{s}$

$S_{i}^{f}: x_{k+1}^{f}=A_{i, 22}^{*} x_{k}^{f}$

with

$A_{i}^{s}=A_{i, 11}^{*}+A_{i, 12}^{*}\left(I_{n_{2}}-A_{i, 22}^{*}\right)^{-1} A_{i, 21}^{*}$

if it exists $L_{i} \in \mathbb{R}^{n_{1} \times n_{2}}$ and $M_{i} \in \mathbb{R}^{n_{2} \times n_{1}}$ matrices satisfying the algebraic equations [6]

$$
\begin{aligned}
& A_{i, 21}^{*}+L_{i}-A_{i, 22}^{*} L_{i}+\mu L_{i}\left[A_{i, 11}^{*}-A_{i, 12}^{*} L_{i}\right]=0 \\
& A_{i, 12}^{*}+M_{i}-M_{i} A_{i, 22}^{*}+\mu\left[A_{i, 11}^{*}-A_{i, 12}^{*} L_{i}\right] M_{i} \\
& \quad-\mu M L_{i} A_{i, 12}^{*}=0
\end{aligned}
$$

$x^{s} \in \mathbb{R}^{n_{1}}$ and $x^{f} \in \mathbb{R}^{n_{2}}$ are, respectively, the slow and the fast subsystems state vectors. Finally, the decoupled discrete nonlinear T-S fuzzy model $S^{d}$ of the original system (3), and the corresponding slow $S^{s}$ and fast $S^{f}$ fuzzy subsystems are respectively given by

$S^{d}:\left[\begin{array}{l}x_{k+1}^{s} \\ x_{k+1}^{f}\end{array}\right]=\left[\begin{array}{cc}\sum_{i=1}^{m} h_{i}\left(I_{n_{1}}+\mu A_{i}^{s}\right) & 0 \\ 0 & \sum_{i=1}^{m} h_{i} A_{i, 22}^{*}\end{array}\right]\left[\begin{array}{l}x_{k}^{s} \\ x_{k}^{f}\end{array}\right]$

$S^{s}: x_{k+1}^{s}=\sum_{i=1}^{m} h_{i}\left(I_{n_{1}}+\mu A_{i}^{s}\right) x_{k}^{s}$

$S^{f}: x_{k+1}^{f}=\sum_{i=1}^{m} h_{i} A_{i, 22}^{*} x_{k}^{f}$

The main objective of the present paper is to provide conditions ensuring the asymptotic stability of the discrete nonlinear T-S fuzzy system (3). We will show that this corresponds in some case to verify the stability conditions of the slow and fast subsystems $(15,16)$ synthesized via singular perturbation technique.

\section{Stability study}

In this section, we recall basic results on stability analysis for T-S fuzzy models based on Lyapunov functions and we formulate the problem. We, then, establish main stability results for the discrete nonlinear original $(1,3)$ and decoupled (14) T-S fuzzy system.

\subsection{Lyapunov functions}

Stability analysis of T-S fuzzy systems has been pursued mainly based on Lyapunov stability. Mainly, three different Lyapunov functions, developed in the literature [31], are introduced below.

\subsubsection{The common (or global) quadratic Lyapunov functions $V(x)=x^{T} P x[38,63]$}

Theorem 1 [38]: The TS fuzzy system (1), or equivalently (3), is globally exponentially stable if there exists a common positive definite matrix such that the following LMIs are satisfied

$A_{i}^{T} P A_{i}-P<0, i \in I$

\subsubsection{The piecewise quadratic Lyapunov functions}

$$
V(x)=\sum_{i=1}^{m} x^{T} P_{i} x
$$

Define $m$ regions in the premise variable space as follows

$D_{i}=\left\{x \mid h_{i}(x)>h_{l}(x) \quad l \in I, \quad l \neq i\right\}, \quad i \in I$

The T-S fuzzy system (3) can be expressed in each local region as

$x_{k+i}=\left(A_{i}+\Delta A_{i}(h)\right) x_{k}, \quad i \in I$

with

$\Delta A_{i}(h)=\sum_{l=1, l \neq i}^{m} h_{l}$

$\Delta A_{i l}, \quad \Delta A_{i l}=A_{l}-A_{i}$

$\left[\Delta A_{i}(h)\right]^{T}\left[\Delta A_{i}(h)\right] \leqslant E_{i A}^{T} E_{i A}$

In addition, define a set $\Omega$ that represents all possible system transitions among regions, that is

$\Omega:=\left\{(i, j) \mid x_{k} \in D_{i}, \quad x_{k+1} \in D_{j}, \quad \forall i, j \in I, i \neq j\right\}$

Theorem 2 [64]: The T-S fuzzy system (1), or equivalently (19), is globally exponentially stable if there exists 
a set of positive-definite matrices $P_{i}, i \in I$, such that the following LMIs are satisfied

$$
\begin{aligned}
& {\left[\begin{array}{cc}
A_{i}^{T} P_{i} A_{i}-P_{i}+E_{i A}^{T} E_{i A} & A_{i}^{T} P_{i} \\
P_{i} A_{i} & -\left(I-P_{i}\right)
\end{array}\right]<0, \quad i \in I} \\
& {\left[\begin{array}{cc}
A_{i}^{T} P_{j} A_{i}-P_{i}+E_{i A}^{T} E_{i A} & A_{i}^{T} P_{j} \\
P_{j} A_{i} & -\left(I-P_{j}\right)
\end{array}\right]<0, \quad i, j \in \Omega}
\end{aligned}
$$

$$
\begin{aligned}
& \text { 3.1.3 The fuzzy (or non-quadratic) Lyapunov functions } \\
& V(x)=\sum_{i=1}^{m} h_{i}(x) x^{T} P_{i} x[65,66]
\end{aligned}
$$

Theorem 3 [65]: The T-S fuzzy system (1), or equivalently (3), is globally exponentially stable if there exists a set of positive-definite matrices $P_{i}, i \in I$ such that the following LMIs are satisfied

$A_{i}^{T} P_{j} A_{i}-P_{j}<0, \quad i \in I, \quad j \in I$

The stability conditions synthesized via the common quadratic Lyapunov functions are very conservative and the introduced approach suffers mainly from few limitations. First, it has been noted that common quadratic Lyapunov functions tend to be conservative, and, might not exist for many complex highly nonlinear systems as shown in [64] and [67]. Second, it appears that a necessary condition, for the existence of this common positive definite matrix, is that all subsystems must be asymptotically stable [38]. Piecewise quadratic Lyapunov functions and fuzzy Lyapunov functions are less conservative but computation cost would be much higher. Vector norms constitute a systematic mean of obtaining comparison systems, which help to overvaluate and analyze nonlinear systems. An adequate choice of the stable overvaluing system may prove the initial system stability. The method is robust and a good choice of the vector norms may allows to obtain conservatism stability conditions [68-72].

In the following, sufficient conditions ensuring asymptotic stability of discrete T-S fuzzy systems (3) with $m$ nonlinear local models (1) are proposed. The aforementioned conditions are developed for original and reduced order decoupled described systems.

\subsection{Proposed stability conditions-main results}

Consider the class of systems $S_{i}$ (1) described by the scalar equation

$\tilde{x}_{k+n}+\sum_{j=1}^{n} a_{i, j}\left(\tilde{x}_{k+n-j}\right) \tilde{x}_{k+n-j}=0, \quad i \in I$

where the corresponding instantaneous characteristic polynomial $P_{S_{i}}(., \lambda)$ is

$P_{S_{i}}(., \lambda)=\lambda^{n}+\sum_{p=1}^{n} a_{i, p}(.) \lambda^{n-p}, \quad i \in I$ and define distinct arbitrary constant parameters $\alpha_{j}, j=$ $1,2, \cdots, n-1$.

For $\alpha_{i} \neq \alpha_{j}, \forall i \neq j$ and $i \in I$, let us introduce to the following notations

$\beta_{j}=\prod_{\substack{k=1 \\ k \neq j}}^{n-1}\left(\alpha_{j}-\alpha_{k}\right)^{-1}, j=1,2, \ldots, n-1$

$\gamma_{j}^{i}()=.-P_{S_{i}}\left(., \alpha_{j}\right) ., \quad j=1,2, \ldots, n-1$

$\delta_{n}^{i}()=.-a_{i, 1}()-.\sum_{p=1}^{n-1} \alpha_{p}$

Let $S$ be a discrete T-S fuzzy system (3), $S_{i}$ a corresponding nonlinear local system of the form (1), $S_{i}^{S}$ the nonlinear decoupled slow local subsystem (9) and $S^{S}$ a nonlinear decoupled slow fuzzy subsystem (15). By applying the BorneGentina practical stability criterion [73-75] to the discrete introduced systems characterized by the Benrejeb arrow form matrix [76-81], we obtain following theorems and corollaries.

Theorem 4 The discrete nonlinear local system $S_{i}$ is asymptotically stable, if there exists constant parameters $\alpha_{i} \in \mathbb{R}$, $\alpha_{i} \neq \alpha_{j} \forall i \neq j$, such that

$\left|\alpha_{i}\right|<1 \quad \forall i=1, \ldots, n-1$

and

$1-\left|\delta_{n}^{i}(\cdot)\right|-\sum_{j=1}^{n-1}\left|\beta_{j}\right|\left|\gamma_{j}^{i}(\cdot)\right|\left(1-\left|\alpha_{j}\right|\right)^{-1}>0$

Proof (Theorem 4) Let us consider the nonlinear local system $S_{i}$ expressed in the Frobenius form as

$\tilde{x}_{k+1}=A_{i}^{F r}\left(\tilde{x}_{n}\right) \tilde{x}_{k}$

with

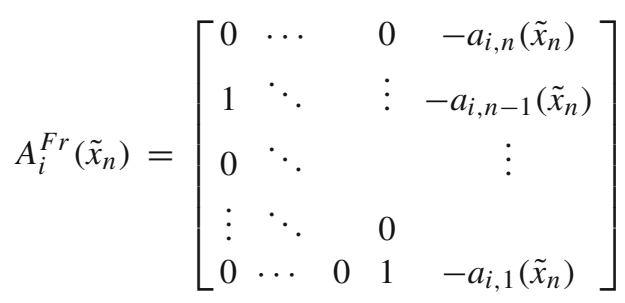

A change of coordinate defined by

$y_{k}=T \tilde{x}_{k}$ 
with $y_{k} \in \mathbb{R}^{n}$ and $T$ an invertible transformation for $\forall \alpha_{i}$, $i=1,2, \cdots, n-1, \alpha_{i} \neq \alpha_{j}$ and $\forall i \neq j$.

$T=\left[\begin{array}{ccccc}0 & 0 & \cdots & 0 & 1 \\ 1 & \alpha_{n-1} & \alpha_{n-1}^{2} & \cdots & \alpha_{n-1}^{n-1} \\ 1 & \alpha_{n-2} & \alpha_{n-2}^{2} & \cdots & \alpha_{n-2}^{n-1} \\ \vdots & \vdots & \vdots & & \vdots \\ 1 & \alpha_{1} & \alpha_{1}^{2} & \cdots & \alpha_{1}^{n-1}\end{array}\right]$

$\operatorname{det}(T)=\prod_{\substack{1 \leq \mathrm{j}<\mathrm{i} \leq \mathrm{n}-1 \\ i \neq j}}\left(\alpha_{i}-\alpha_{j}\right)$

leads to the following state space description

$y_{k+1}=G_{i}$ (.) $y_{k}$

Allowing the synthesis of sufficient stability conditions easy to test, the new instantaneous characteristic matrix $G_{i}($.$) is$ chosen to be in the arrow form [76-81], Appendix 2, as following

$G_{i}()=.T A_{i}^{F r}(.) T^{-1}=\left[\begin{array}{llll}\delta_{n}^{i}(.) & \beta_{1} \cdots & \beta_{n-1} \\ \gamma_{1}^{i}(.) & \alpha_{1} & & \\ \vdots & & \ddots & \\ \gamma_{n-1}^{i}(.) & & & \alpha_{n-1}\end{array}\right]$

where $\beta_{i}, \gamma_{j}^{i}, \delta_{n}^{i}$ and $\alpha_{i}, i=1,2, \ldots, n-1$, are defined by the relations (27-29). A pseudo-overvaluing matrix $M\left(G_{i}(\cdot)\right)$ of the system (37), corresponding to the use of the vector norm (Appendix 1) $p(y)$ such that

$p(y)=\left[\left|y_{1}\right|,\left|y_{2}\right|, \ldots,\left|y_{n}\right|\right]^{T}$

$y=\left[y_{1}, y_{2}, \ldots, y_{n}\right]^{T}$, for the stability study, can be obtained from the inequality

$p\left(y_{k+1}\right) \leqslant M\left(G_{i}(\cdot)\right) p\left(y_{k}\right)$

satisfied for each corresponding component; that leads to the following comparison system

$z_{k+1}=M\left(G_{i}(\cdot)\right) z_{k}$

with

$M\left(G_{i}().\right)=\left[\begin{array}{llll}\left|\delta_{n}^{i}(\cdot)\right| & \left|\beta_{1}\right| & \cdots & \left|\beta_{n-1}\right| \\ \left|\gamma_{1}^{i}(\cdot)\right| & \left|\alpha_{1}\right| & \\ \vdots & & \ddots & \\ \left|\gamma_{n-1}^{i}(\cdot)\right| & & \left|\alpha_{n-1}\right|\end{array}\right]$

such as $z_{0}=p\left(y_{0}\right)$. If the nonlinearities of the comparison nonlinear system (41) are isolated in one row of $M\left(G_{i}(\cdot)\right)$, the verification of the Kotelyanski condition (Appendix 1) enables to conclude to the stability of the original system characterized by $G_{i}(\cdot)$ [74]. It comes the following sufficient asymptotic stability condition of the original system $S_{i}$

$$
\left(I_{n}-M\left(G_{i}(\cdot)\right)\right)\left(\begin{array}{llll}
1 & 2 & \ldots j \\
1 & 2 & \ldots & j
\end{array}\right)>0 j=1, \ldots, n
$$

This ends the proof of Theorem 4.
Theorem 5 The discrete nonlinear decoupled local system $S_{i}^{d}(8)$ is asymptotically stable if there exists $\alpha_{i} \in \mathbb{R}, \alpha_{i} \neq \alpha_{j}$ $\forall i \neq j$, such that

$$
\left|\alpha_{i}\right|<1 \forall i=1, \ldots, n-1
$$

and

$$
\begin{aligned}
& -\left|\delta_{n}^{i}(\cdot)+\sum_{j=n_{1}}^{n-1} \beta_{j} \gamma_{j}^{i}(\cdot)\left(1-\alpha_{j}\right)^{-1}\right| \\
& -\sum_{j=1}^{n_{1}-1}\left|\beta_{j}\right|\left|\gamma_{j}^{i}(\cdot)\right|\left(1-\left|\alpha_{j}\right|\right)^{-1}>0
\end{aligned}
$$

Proof (Theorem 5) Note that the satisfaction of the conditions (30), i.e. $\left|\alpha_{i}\right|<1, i=1, \ldots, n-1$, means that the fast system characterized by a diagonal matrix $\left\{\alpha_{i}\right\}, i=n_{1}, \ldots, n-1$ is stable. Conditions $\left|\alpha_{i}\right|<$ $1, i=1, \ldots, n_{1}-1$, are necessary to satisfy the reduced slow subsystem stability. In order to synthesize the stability conditions of the two-time-scale decoupled system $S_{i}$, we, consider the transformed nonlinear system states (38). Resulting $A_{i, 11}, A_{i, 12}, A_{i, 21}$ and $A_{i, 22}$ matrices are then in the form (46) where the matrix $A_{i, 11}$ is candidate to characterize the slow subsystem of (1) and $A_{i, 22}$ the fast one.

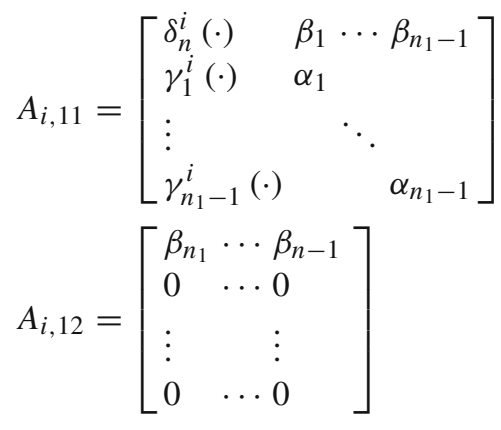

$A_{i, 21}=\left[\begin{array}{llll}\gamma_{n_{1}}^{i}(\cdot) & 0 & \cdots & 0 \\ \vdots & \vdots & & \vdots \\ \gamma_{n-1}^{i}(\cdot) & 0 & \cdots & 0\end{array}\right]$

$A_{i, 22}=\left[\begin{array}{lll}\alpha_{n_{1}} & & 0 \\ & \ddots & \\ 0 & & \alpha_{n-1}\end{array}\right]$

Arbitrary constant parameters $\alpha_{i}, i=n_{1}, \ldots, n-1$, are chosen in concordance with the estimation of the dynamics that what we consider physically fast for the studied system. Substituting the relations (46), (6) and (11) into (9) and (10), yields to following discrete slow and fast subsystems, respectively

$x_{k+1}^{s}=F_{i}^{s}$ (.) $x_{k}^{s}$

$x_{k+1}^{f}=F_{i}^{f} x_{k}^{f}$

and then corresponding comparison systems, respectively

$y_{k+1}^{s}=M\left(F_{i}^{s}(\cdot)\right) y_{k}^{s}$

$y_{k+1}^{f}=M\left(F_{i}^{f}\right) y_{k}^{f}$ 
where $F_{i}^{s} \in \mathbb{R}^{n_{1} \times n_{1}}$ and $F_{i}^{f} \in \mathbb{R}^{n_{2} \times n_{2}}$ are given by

$F_{i}^{s}()=.\left[\begin{array}{l}\delta_{n}^{i}(\cdot) \\ +\sum_{j=n_{1}}^{n-1} \beta_{j} \gamma_{j}^{i}(\cdot)\left(1-\alpha_{j}\right)^{-1} \\ \gamma_{1}^{i}(\cdot) \\ \vdots \\ \gamma_{n_{1}-1}^{i}(\cdot)\end{array}\right.$ $\left.\begin{array}{ccc} & & \\ \beta_{1} \ldots & \beta_{n_{1}-1} \\ \alpha_{1} & & \\ & \ddots & \\ & & \alpha_{n_{1-1}}\end{array}\right]$

(51)

$F_{i}^{f}=\left[\begin{array}{lll}\alpha_{n_{1}} & & \\ & \ddots & \\ & & \alpha_{n-1}\end{array}\right]$

and $M\left(F_{i}^{s}(\cdot)\right)$ and $M\left(F_{i}^{f}\right)$ are respectively the pseudoovervaluing matrices of the slow and fast subsystems (9) and (10), corresponding to the use of the vector norm (39). By applying the practical Borne-Gentina stability criterion [73-75] to the comparison systems (49) and (50) of (47) and (48), we deduce the stability conditions of the decoupled discrete systems $S_{i}^{d}(8)$. The Theorem 5 is then proved.

Corollary 1 If the discrete nonlinear system $S_{i}(1)$ is asymptotically stable, i.e. the following conditions are satisfied

(i) $\exists \varepsilon>0$ and $\alpha_{j} \in \mathbb{R}, 0<\alpha_{j}<1, \alpha_{j} \neq \alpha_{k}, \forall j \neq$ $k, j, k=1, \cdots, n-1$ such that

$$
\left\{\begin{array}{l}
\delta_{n}^{i}(.)>0 \\
\gamma_{j}^{i}(.) \beta_{j}>0 \quad \forall j=1, \ldots, n-1
\end{array}\right.
$$

(ii)

$$
\begin{aligned}
\left.P_{S_{i}}(., \lambda)\right|_{\lambda=1} \geqslant & \varepsilon>0, \quad \text { i.e. } \\
& 1+\sum_{p=1}^{n} a_{i, p}(.)>0, \quad i \in I
\end{aligned}
$$

then, the corresponding decoupled nonlinear system $S_{i}^{d}$ is asymptotically stable.

Proof (Corollary 1) By considering conditions (i) of the Corollary 1, and substituting relations (27-29) in (31), the stability condition (31) of the discrete nonlinear local system $S_{i}$ (1) becomes

$$
\begin{aligned}
1 & +a_{i, 1}(.)+\sum_{p=1}^{n-1} \alpha_{p} \\
+ & \sum_{p=1}^{n-1} \frac{1}{1-\alpha_{p}}\left(\frac{\left(\lambda-\alpha_{p}\right) P_{S_{i}}(., \lambda)}{Q(\lambda)}\right)_{\lambda=\alpha_{p}}>0
\end{aligned}
$$

with

$$
Q(\lambda)=\prod_{p=1}^{n-1}\left(\lambda-\alpha_{p}\right)
$$

To deduce the stability conditions of the decoupled system $S_{i}^{d}(8)$, let us first observe that

$$
\begin{aligned}
\frac{P_{S_{i}}(., \lambda)}{Q(\lambda)}= & \lambda+a_{i, 1}(.)+\sum_{p=1}^{n-1} \alpha_{p} \\
& +\sum_{p=1}^{n-1} \frac{1}{\lambda-\alpha_{p}}\left(\frac{\left(\lambda-\alpha_{p}\right) P_{S_{i}}(., \lambda)}{Q(\lambda)}\right)_{\lambda=\alpha_{p}}
\end{aligned}
$$

It, then, follows that the developed stability condition (55) is equivalent to

$$
\left.\frac{P_{S_{i}}(., \lambda)}{Q(\lambda)}\right|_{\lambda=1}>0
$$

or

$$
\left.P_{S_{i}}(., \lambda)\right|_{\lambda=1}>0
$$

which yields

$1+\sum_{p=1}^{n} a_{i, p}()>0,. \quad i \in I$

and constitutes a verification case of the validity of the linear Aizerman conjecture [82,83]. These conditions, associated to aggregation techniques based on the use of vector norms, have led to stability domains for a class of Lure-Postnikov systems whereas, for example, Popov stability criterion use failed. The proof is easily completed by substituting the conditions (i) in stability condition (45) of the discrete nonlinear decoupled system $S_{i}^{d}(8)$.

Corollary 2 If the discrete nonlinear decoupled system $S_{i}^{d}(8)$ is asymptotically stable, i. e. the following conditions are satisfied

(i) $\exists \varepsilon>0$ and $\alpha_{j} \in \mathbb{R}, \alpha_{j} \neq \alpha_{k}, \forall j \neq k ; j, k=$ $1, \ldots, n-1$, and $0<\alpha_{j}<1 j=1, \ldots, n_{1}-1$ such that

$$
\left\{\begin{array}{l}
\delta_{n}^{i}(\cdot)+\sum_{j=n_{1}}^{n-1} \beta_{j} \gamma_{j}^{i}(\cdot)\left(1-\alpha_{j}\right)^{-1}>0 \\
\gamma_{n}^{i}(.) \beta_{j}>0 \quad \forall j=1, \ldots, n_{1}-1
\end{array}\right.
$$

(ii)

$$
\begin{aligned}
\left.P_{S_{i}}(., \lambda)\right|_{\lambda=1} \geqslant & \varepsilon>0 \quad \text { i.e. } \\
& 1+\sum_{p=1}^{n} a_{i, p}(.)>0, \quad i \in I
\end{aligned}
$$

then, the original discrete nonlinear local system $S_{i}(1)$ is asymptotically stable if the following additional conditions 
are satisfied

$$
\begin{cases}0<\alpha_{j}<1 & \forall j=n_{1}, \ldots, n-1 \\ \delta_{n}^{i}(.)>0 & \\ \gamma_{j}^{i}(.) \beta_{j}>0 & \forall j=n_{1}, \ldots, n-1\end{cases}
$$

Proof (Corollary 2) Conditions (i) imply stability condition (ii) as demonstrated in Corollary 1 proof. Indeed if (62) are satisfied, then it is easy to see that stability conditions (30-31) of the original discrete nonlinear system $S_{i}(1)$ are verified.

Theorem 6 The discrete nonlinear T-S fuzzy system $S$ (3) is asymptotically stable if there exist constant parameters $\alpha_{i} \in \mathbb{R}, \alpha_{i} \neq \alpha_{j} \forall i \neq j$, such that $\forall x \in D$.

$\left|\alpha_{i}\right|<1 \forall i=1, \ldots, n-1$

and

$1-\left|\sum_{i=1}^{m} h_{i} \delta_{n}^{i}(\cdot)\right|-\sum_{j=1}^{n-1}\left|\beta_{j}\right|\left|\sum_{i=1}^{m} h_{i} \gamma_{j}^{i}(\cdot)\right|\left(1-\left|\alpha_{j}\right|\right)^{-1}>0$

If $D=\mathbb{R}^{n}$, the stability is global.

Proof (Theorem 6) Based on the state transformed form of the local nonlinear systems (37), the discrete T-S fuzzy model (3) can be rewritten as

$y_{k+1}=G(.) y_{k}$

where $G($.$) is given by$

$G()=.\sum_{i=1}^{m} h_{i} G_{i}($.

It follows that

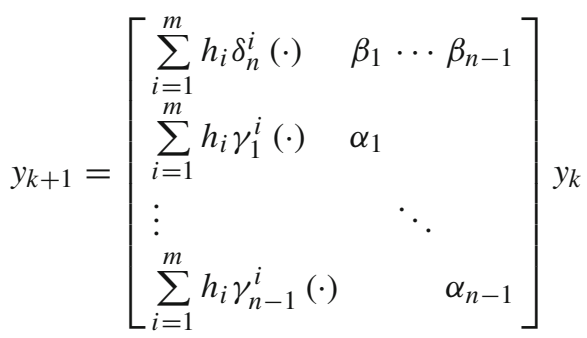

Now, by introducing the comparison system

$z_{k+1}=M(G(\cdot)) z_{k}$

where $M(G(\cdot))$ is the pseudo-overvaluing matrix of (3), corresponding to the use of the vector norm (39). By applying the practical Borne-Gentina criterion [73-75] to the comparison system (68), we deduce the stability conditions of the nonlinear discrete T-S fuzzy system (3). This ends the Theorem 6 proof.
Theorem 7 The discrete nonlinear decoupled T-S fuzzy system $S^{d}$ (14) is asymptotically stable if there exists $\alpha_{i} \in$ $\mathbb{R}, \alpha_{i} \neq \alpha_{j} \forall i \neq j$, such that

$\left|\alpha_{i}\right|<1 \quad \forall i=1, \cdots, n-1$

and

$$
\begin{aligned}
1 & -\left|\sum_{i=1}^{m} h_{i} \delta_{n}^{i}(\cdot)+\sum_{j=n_{1}}^{n-1} \beta_{j} \sum_{i=1}^{m} h_{i} \gamma_{j}^{i}(\cdot)\left(1-\alpha_{j}\right)^{-1}\right| \\
& -\sum_{j=1}^{n_{1}-1}\left|\beta_{j}\right|\left|\sum_{i=1}^{m} h_{i} \gamma_{j}^{i}(\cdot)\right|\left(1-\left|\alpha_{j}\right|\right)^{-1}>0
\end{aligned}
$$

Proof (Theorem 7) By substituting relations (6) and (11) in (15) and (16) where matrices $A_{i, 11}, A_{i, 12}, A_{i, 21}$ and $A_{i, 22}$ are represented in the arrow form (46), we obtain the following slow and fast reduced order discrete T-S fuzzy systems, respectively

$x_{k+1}^{s}=F^{s}$ (.) $x_{k}^{s}$

$x_{k+1}^{f}=F^{f} x_{k}^{f}$

and then comparison systems, respectively

$y_{k+1}^{s}=M\left(F^{s}(\cdot)\right) y_{k}^{s}$

$y_{k+1}^{f}=M\left(F^{f}\right) y_{k}^{f}$

$F^{s}(\cdot) \in \mathbb{R}^{n_{1} \times n_{1}}$ and $F^{f} \in \mathbb{R}^{n_{2} \times n_{2}}$ are respectively given by

$F^{S}=\left[\begin{array}{llll}\sum_{i=1}^{m} h_{i} \delta_{n}^{i}(\cdot) & & & \\ +\sum_{j=n_{1}}^{n-1} \beta_{j} \sum_{i=1}^{m} h_{i} \gamma_{j}^{i}(\cdot)\left(1-\alpha_{j}\right)^{-1} & \beta_{1} & \ldots & \beta_{n_{1}-1} \\ \sum_{i=1}^{m} h_{i} \gamma_{1}^{i}(\cdot) & \alpha_{1} & & \\ \vdots & & \ddots & \\ \sum_{i=1}^{m} h_{i} \gamma_{n_{1}-1}^{i}(\cdot) & & \alpha_{n_{1}-1}\end{array}\right]$

$F^{f}=\left[\begin{array}{lll}\alpha_{n_{1}} & & \\ & \ddots & \\ & & \alpha_{n-1}\end{array}\right]$

and $M\left(F^{s}(\cdot)\right)$ and $M\left(F^{f}\right)$ are respectively the pseudoovervaluing matrices of the slow and fast subsystems (15) and (16), corresponding to the use of the vector norm (39). Stability condition for the discrete decoupled system (14) is synthesized by the application of Borne and Gentina stability criterion, that completes the proof.

A generalized form of Corollary 1 and 2 can be developed for original T-S fuzzy system (3) and the decoupled T-S fuzzy system (14) by substituting $a_{i, j}(),. \delta_{n}^{i}(),. \gamma_{j}^{i}$ (.) 
and $P_{S_{i}}(., \lambda)$ respectively by $a^{\prime}{ }_{j}(),. \delta^{\prime}{ }_{n}(),. \gamma^{\prime}{ }_{j}($.$) and$ $P_{S}^{\prime}(., \lambda)$ such that

$a_{j}^{\prime}()=.\sum_{i=1}^{m} h_{i} a_{i, j}($.

$\delta_{n}^{\prime}()=.\sum_{i=1}^{m} h_{i} \delta_{n}^{i}($.

$\gamma_{j}^{\prime}()=.\sum_{i=1}^{m} h_{i} \gamma_{j}^{i}($.

$P_{S}^{\prime}(., \lambda)=\lambda^{n}+\sum_{j=1}^{n} a_{j}^{\prime}(.) \lambda^{n-j}$

Corollary 3 If the nonlinear discrete T-S fuzzy system $S$ (3) is asymptotically stable, i.e. the following conditions are satisfied

(i) $\exists \varepsilon>0$ and $\alpha_{j} \in \mathbb{R}, 0<\alpha_{j}<1, \alpha_{j} \neq \alpha_{k}, \forall j \neq$ $k ; j, k=1, \ldots, n-1$ such that

$$
\left\{\begin{array}{l}
\delta_{n}^{\prime}(.)>0 \\
\gamma^{\prime}{ }_{j}(.) \beta_{j}>0 \quad \forall j=1, \ldots, n-1
\end{array}\right.
$$

(ii)

$$
\left.P_{S}^{\prime}(., \lambda)\right|_{\lambda=1} \geqslant \varepsilon>0
$$

then, the corresponding decoupled T-S system (14) is asymptotically stable.

Corollary 4 If the nonlinear discrete decoupled T-S fuzzy system (14) is asymptotically stable, i.e. the following conditions are satisfied

(i) $\exists \varepsilon>0$ and $\alpha_{j} \in \mathbb{R}, \alpha_{j} \neq \alpha_{k}, \forall j \neq k ; j, k=$ $1, \ldots, n-1$, and $0<\alpha_{j}<1 j=1, \ldots, n_{1}-1$ such that

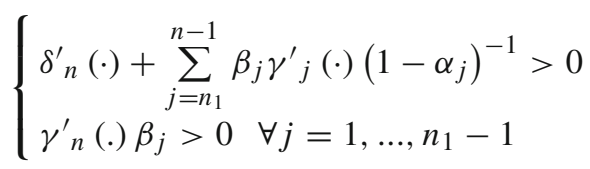

(ii)

$$
\begin{aligned}
&\left.P_{S}^{\prime}(., \lambda)\right|_{\lambda=1} \geqslant \varepsilon>0 \text { i.e. } \\
& 1+\sum_{p=1}^{n} a_{p}^{\prime}(.)>0
\end{aligned}
$$

then, the original discrete nonlinear T-S fuzzy system (3) is asymptotically stable if the following additional conditions are satisfied

$$
\begin{cases}0<\alpha_{j}<1 & \forall j=n_{1}, \ldots, n-1 \\ \delta^{\prime}{ }_{n}(.)>0 & \forall j=n_{1}, \ldots, n-1 \\ \gamma^{\prime}{ }_{j}(.) \beta_{j}>0 & \end{cases}
$$

\section{Example: case of third order system}

Consider a T-S fuzzy model based system such that the consequence of the rule $R_{i}$ is in the form

$x_{k+1}=A_{i}(.) x_{k}, \quad i=1,2$

$A_{i}()=.\left[\begin{array}{rrr}0 & 0 & -1,19.10^{-6} f_{i}(.) \\ 1 & 0 & -0,13+0,23.10^{-1} f_{i}(.) \\ 0 & 1 & 1,13-1,92 f_{i}(.)\end{array}\right], \quad i=1,2$

The local systems (86) with the characteristic matrix $G_{i}$ (.) and the synthesized T-S fuzzy system with $G($.$) can be,$ respectively, expressed in the arrow form as following

$$
\begin{aligned}
G_{i}(.)= & {\left[\begin{array}{lcc}
0,14-0,19 f_{i}(.) & 1,20-1,20 \\
0,69.10^{-1}-0,14 f_{i}(.) & 0,90 & 0 \\
-0,32.10^{-2}-0,37.10^{-3} f_{i}(.) & 0 & 0,10
\end{array}\right] } \\
& i=1,2
\end{aligned}
$$

$$
\begin{aligned}
& G(.)= \\
& {\left[\begin{array}{lcc}
0,14-0,038 f_{1}(.)-0,152 f_{2}(.) & 1,20 & -1,20 \\
0,69.10^{-1}-0,028 f_{1}(.)-0,112 f_{2}(.) & 0,90 & 0 \\
-0,32.10^{-2}-0,74.10^{-4} f_{1}(.) & 0 & 0,10
\end{array}\right]}
\end{aligned}
$$

for $\alpha_{1}=0.9$ and $\alpha_{2}=0.1$ satisfying (30), $h 1=0.2, h 2=$ 0.8 and $\mu=0.1$. The decoupled slow and fast subsystems for the local nonlinear systems (86) are given respectively by

$F_{i}^{s}()=.\left[\begin{array}{ll}0,14-0,19 f_{i}(.) & 1,20 \\ 0,69.10^{-1}-0,14 f_{i}(.) & 0,90\end{array}\right] i=1,2$

$F_{i}^{f}=0,10$

and for the T-S fuzzy system (89) respectively by

$F^{s}()=.\left[\begin{array}{ll}0,14-0,038 f_{1}(.)-0,152 f_{2}(.) & 1,20 \\ 0,69.10^{-1}-0,028 f_{1}(.)-0,112 f_{2}(.) & 0,90\end{array}\right]$

$F^{f}=0,10$

In the following, we determine the stability domains of original and decoupled described systems. For the chosen $\alpha_{1}$ and $\alpha_{2}$, synthesized stability condition of the discrete T-S fuzzy 
Table 1 Stability domain of the original T-S fuzzy system (89)

\begin{tabular}{ll}
\hline$f_{1}($.$) variation$ & $f_{2}($.$) variation$ \\
\hline $0.464 \leqslant f_{1}<3.046$ & $-0.258+0.037 f_{1}<f_{2}<1.312-0.478 f_{1}$ \\
$0.135 \leqslant f_{1}<0.464$ & $-0.018-0.478 f_{1}<f_{2}<1.312-0.478 f_{1}$ \\
$-0.193<f_{1}<0.135$ & $-0.018-0.478 f_{1}<f_{2}<1.312-0.478 f_{1}$ \\
$-2.776<f_{1} \leqslant-0.193$ & $-0.018-0.478 f_{1}<f_{2}<1.412+0.036 f_{1}$ \\
else & $\emptyset$ \\
\hline
\end{tabular}

Table 2 Stability domain of the decoupled T-S fuzzy system (91)

\begin{tabular}{ll}
\hline$f_{1}($.$) variation$ & $f_{2}($.$) variation$ \\
\hline $0.466 \leqslant f_{1}<3.059$ & $-0.261+0.037 f_{1}<f_{2}<1.315-0.478 f_{1}$ \\
$0.135 \leqslant f_{1}<0.466$ & $-0.021-0.478 f_{1}<f_{2}<1.315-0.478 f_{1}$ \\
$-0.195<f_{1}<0.135$ & $-0.021-0.478 f_{1}<f_{2}<1.315-0.478 f_{1}$ \\
$-2.788<f_{1} \leqslant-0.195$ & $-0.021-0.478 f_{1}<f_{2}<1.416+0.036 f_{1}$ \\
else & $\emptyset$ \\
\hline
\end{tabular}

system (89) deduced from Theorem 6, is the following

$1-\left|0,14-0,038 f_{1}-0,152 f_{2}\right|$

$-12\left|0,69.10^{-1}-0,028 f_{1}-0,112 f_{2}\right|$

$-1.33\left|-0,32.10^{-2}-0,74.10^{-4} f_{1}-0,296.10^{-4} f_{2}\right|>0$

Using condition (92), system (89) is stable if nonlinear functions $f_{1}($.$) and f_{2}($.$) are, respectively, within the following$ limits, given in Table 1. Furthermore, applying Theorem 4 to the nonlinear local system (86) yields

$-0.0148<f_{i}()<.1.0498 \quad i=1,2$

Now, for the synthesized decoupled discrete T-S fuzzy system (91), sufficient stability condition issued from Theorem 7 , is given by

$$
\begin{aligned}
& 1-\left|0,14-0,038 f_{1}(.)-0,152 f_{2}(.)\right| \\
& \quad-12\left|0,69.10^{-1}-0,028 f_{1}(.)-0,112 f_{2}(.)\right|>0
\end{aligned}
$$

Deriving additional conditions on $f_{1}\left(\right.$.) and $f_{2}$ (.) for the existence of a solution to stability condition (94), results of Table 2 are obtained. Moreover, according to Theorem 5, the nonlinear local systems (90) is stable for

$$
-0.0171<f_{i}(.)<1.0524 \quad i=1,2
$$

Figure 1 illustrates the stability domains $D_{1}, D_{2}, D_{3}$ and $D_{4}$ associated respectively to the original discrete T-S fuzzy system (77), the decoupled T-S fuzzy system (91), the nonlinear local model (86) and the decoupled nonlinear local model (90). As shown, the stability domain of the decoupled

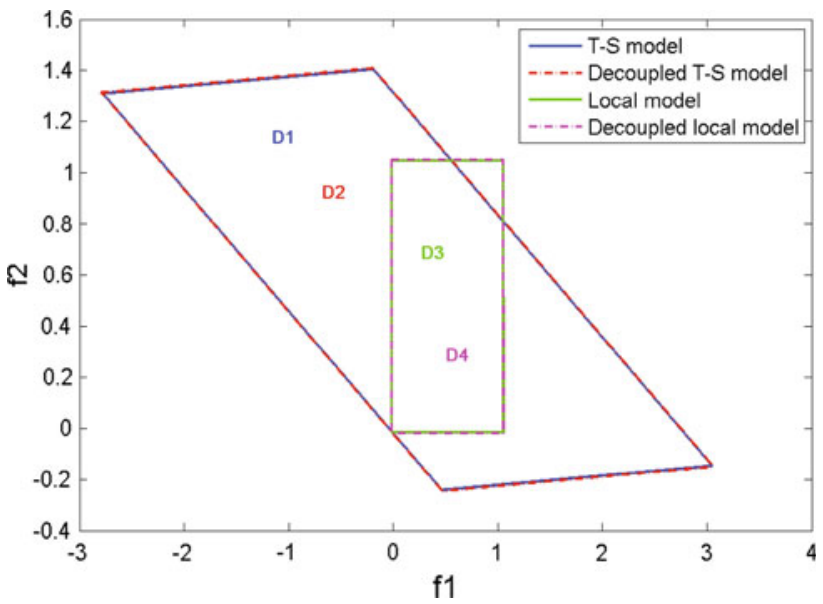

Fig. 1 Stability domains

systems (90) and (91)are, respectively, very close to the original ones (86) and (89). Furthermore, one can see that the stability conditions (30-31) and (44-45) of local systems are conservative and induce smaller stability domains. Discrete T-S fuzzy and local models have the common restricted stability domain $D_{5}=D_{1} \cap D_{2} \cap D_{3} \cap D_{4} . D_{5}$ is smaller than the common estimated stability region of local systems; the stability of each local model does not ensure the stability of the global system.

\section{Conclusion}

In this paper, we have investigated the stability problem of singular T-S fuzzy systems under the discrete-time framework. By using the arrow matrix form and Borne and Gentina criterion, sufficient stability conditions for of the reduced 
order decoupled T-S fuzzy system, as well as the original T-S fuzzy system are derived. Supplementary stability conditions are synthesized to ensure a common stability domain for the original and the decoupled T-S fuzzy system. In the simulation, an illustrative example demonstrated that obtained results are less conservative than existing ones.

\section{Appendix 1}

Definition 1 (Vector Norm [84,85]) Let $E=\mathbb{R}^{n}$ be a vector space and $E_{1}, E_{2}, \cdots, E_{k}$ subspaces of $E$ which verify: $E=$ $E_{1} \cup E_{2} \cup \cdots \cup E_{k}$. Let $x \in E$ be an $n$ vector defined on $E$ with a projection in the subspace $E_{i}$ denoted by $x_{i}, x_{i}=$ $P_{i} x$, where $P_{i}$ is a projection operator from $E$ into $E_{i}, p_{i}$ is a scalar norm $(i=1, \cdots, k)$ defined on the subspace $E_{i}$ and $p$ denotes the vector norm of dimension $k$ and with $i$ th component, $p_{i}(x): \mathbb{R}^{n} \rightarrow \mathbb{R}_{+}^{k}$, where $p_{i}\left(x_{i}\right)$ is a scalar norm of $x_{i}$.

Lemma 1 (Kotelyanski [86,87]) The real parts of the eigenvalues of matrix A, with non negative off diagonal elements, are less than a real number $\mu$ if and only if all those of matrix $M=\mu I_{n}-A$ are positive, with $I_{n}$ the $n$ identity matrix.

When successive principal minors of matrix $(-A)$ are positive, Kotelyanski lemma permits to conclude on stability property of the system characterized by A.

Theorem 8 (Borne and Gentina practical stability criterion $[73,75])$ Let consider the nonlinear discrete system

$z_{k+1}=A(.) z_{k}$

and the overvaluing matrix

$M(A(\cdot))=\left\{\left|a_{j, k}\right|\right\}, \forall j, k=1, \cdots, n$

If the nonlinearities are isolated in either one row or one column of $M(A(\cdot))$, the verification of the Kotelyanski condition enables to conclude to the stability of the original system characterized by $A(\cdot)$. Kotelyanski lemma applied to the overvaluing matrix obtained by the use of the regular vector norm:

$p_{z}(k)=\left[\left|z_{1}(k)\right|,\left|z_{2}(k)\right|, \ldots,\left|z_{n}(k)\right|\right]^{T}$

with $z(k)=\left[z_{1}(k), z_{2}(k), \ldots, z_{n}(k)\right]^{T}$, leads to the following sufficient conditions of asymptotic stability of original system

$\left(I_{n}-M(A(\cdot))\right)\left(\begin{array}{llll}1 & 2 & \ldots & j \\ 1 & 2 & \ldots & j\end{array}\right)>0 j=1, \ldots, n$

This criterion is useful for the stability study of complex and large scale systems, such that the necessary condition of its application is satisfied or if the system parameters identification is imprecise. The Borne et Gentina practical criterion applied to discrete systems generalizes the Kotelyanski lemma for non linear systems and defines large classes of systems for which the linear conjecture can be applied, either for the original system or for its comparison system.

\section{Appendix 2: On arrow form matrix}

Let us consider the observable nonlinear system

$$
\begin{aligned}
& z_{k+1}=A(.) z_{k} \\
& A(.)=\left[\begin{array}{llll}
0 & \cdots & 0 & -a_{n}(.) \\
1 & 0 & \vdots & -a_{n-1}(.) \\
0 & \ddots & 0 & \vdots \\
0 & 0 & 1 & -a_{1}(.)
\end{array}\right]
\end{aligned}
$$

$a_{i}($.$) are the instantaneous characteristic polynomial P_{A}(., \lambda)$ coefficients of $A($.$) , such that$

$$
P_{A}(., \lambda)=\lambda^{n}+\sum_{i=1}^{n} a_{i}(.) \lambda^{n-i}
$$

A change of base, defined by

$\hat{z}_{k}=T z_{k}$

$$
T=\left[\begin{array}{lllll}
0 & 0 & \cdots & 0 & 1 \\
1 & \alpha_{n-1} & \alpha_{n-1}^{2} & \cdots & \alpha_{n-1}^{n-1} \\
1 & \alpha_{n-2} & \alpha_{n-2}^{2} & \cdots & \alpha_{n-2}^{n-1} \\
\vdots & \vdots & \vdots & \vdots & \vdots \\
1 & \alpha_{1} & \alpha_{1}^{2} & \cdots & \alpha_{1}^{n-1}
\end{array}\right]
$$

where $\alpha_{j}, j=1,2, \cdots, n-1$ are distinct arbitrary constant parameters, allows the new state matrix, denoted by $F($.$) , to$ be in arrow form $[76,80]$

$$
F(.)=T A(.) T^{-1}=\left[\begin{array}{llll}
\delta_{n}(.) & \beta_{1} & \cdots & \beta_{n-1} \\
\gamma_{1}(.) & \alpha_{1} & & \\
\vdots & & \ddots & \\
\gamma_{n-1}(.) & & & \alpha_{n-1}
\end{array}\right]
$$

with

$$
\begin{aligned}
& \beta_{j}=\prod_{\substack{k=1 \\
k \neq j}}^{n-1}\left(\alpha_{j}-\alpha_{k}\right)^{-1}, \forall j=1,2, \ldots, n-1 \\
& \delta_{j}(.)=-P_{A}\left(., \alpha_{j}\right), \forall j=1,2, \ldots, n-1 \\
& \delta_{n}(.)=-a_{1}(.)-\sum_{i=1}^{n-1} \alpha_{i}
\end{aligned}
$$

This particular form allows having the non-constant elements of the free state matrix isolated in the first column, which 
makes it possible to established a stability criterion for the nonlinear system in the multimodel approach.

With the use of Benrejeb arrow form matrices for characteristic matrices, and of vector norms as Lyapunov functions, the criterion defines large classes of systems for which the Aizerman conjecture to a comparison system is satisfied.

\section{References}

1. Saksena VR, O'Reilly J, Kokotovic PV (1984) Singular perturbations and time-scale methods in control theory: survey 1976-1983. Automatica 20:273-293

2. Kokotovic PV, Khalil HK, O'Reilly J (1986) Singular perturbation methods in control: analysis and design. Academic, London

3. Shao PZH, Sawan ME (1993) Robust stability of singularly perturbed systems. Int J Control 58:1469-1476

4. Naidu DS (2002) Singular perturbations and time scales in control theory and applications: an overview, Dynamics of Continuous. Discret Impuls Syst 9:233-278

5. Li THS, Chiou JS, Kung FC (1999) Stability bounds of singularly perturbed discrete systems. IEEE Trans Autom Control 44:19341938

6. Litkouhi B, Khalil HK (1985) Multirate and composite control of two-time-scale discrete-time systems. IEEE Trans Autom Control 30:645-651

7. Ghosh R, Sen S, Datta KB (1999) Method for evaluating stability bounds for discrete time singularly perturbed systems. IEE Proc Control Theory Appl 146:227-233

8. Kafri WS, Abed EH (1996) Stability analysis of discrete-time singularly perturbed systems. IEEE Trans Circuits Syst -I: Fundam Theory Appl 43:848-850

9. Liyu C, Schwartz HM (2004) Complementary results on the stability bounds of singularly perturbed systems. IEEE Trans Autom Control 49:2017-2021

10. Shao ZH (2004) Robust stability of two-time-scale systems with nonlinear uncertainties. IEEE Trans Autom Control 49:258-261

11. Loescharataramdee C, Edwin Sawan M (1999) Stability robustness bounds of discrete two-time-scale systems. J Frank1 Inst 336:973981

12. Grujic LT (1981) Uniform asymptotic stability of nonlinear singularly perturbed and large scale systems. Int J Control 33:481-504

13. Chow JH (1978) Asymptotic stability of a class of nonlinear singularly perturbed systems. J Frankl Inst 305:275-281

14. Khalil HK (1981) Asymptotic stability of nonlinear multiparameter singularly perturbed systems. Automatica 17:797-804

15. Saberi A, Khalil HK (1984) Quadratic type Lyapunov functions for singularly perturbed systems. IEEE Trans Autom Control 29:542550

16. Khorasani K, Pai MA (1985) Asymptotic stability of nonlinear singularly perturbed systems using higher order corrections. Automatica 21:717-727

17. Bouyekhf R, El Moudni A (1997) On analysis of discrete singularly perturbed non-linear systems: application to the study of stability properties. J Frankl Inst 334:199-212

18. Dong J, Yang GH (2008) H $\infty$ control for fast sampling discretetime singularly perturbed systems. Automatica 44:1385-1393

19. Malloci I, Daafouz J, Iung C (2010) Stability and stabilization of two time scale switched systems in discrete time. IEEE Trans Autom Control 55:1434-1438

20. Park KS, Lim JT (2011) Stability analysis of nonstandard nonlinear singularly perturbed discrete systems. IEEE Trans Circuits Syst-II: Express Briefs 58:309-313
21. Park KS, Cho YJ, Kim YJ, Lim JT (2011) Stability analysis of uncertain nonlinear singularly perturbed discrete systems, 2011 Third International Conference on Computational Intelligence. Modelling and Simulation, Langkawi, pp $153-158$

22. Son JW, Lim JT (2006) Robust stability of nonlinear singularly perturbed system with uncertainties. IEE Proc Control Theory Appl 153:104-110

23. Wang HJ, Xue AK, Lu RQ (2009) Absolute stability criteria for a class of nonlinear singular system with time delay. Nonlinear Anal 70:621-630

24. Mamdani EH (1974) Applications of fuzzy algorithms for control of simple dynamic plants. Proc Inst Electr Eng 121:1585-1588

25. Zadeh LA (1965) Fuzzy sets. Inf Control 8:338-353

26. Acampora G (2011) A TSK Neuro-Fuzzy Approach for Modeling Highly Dynamic Systems, 2011 IEEE International Conference on Fuzzy Systems, Taipei, June 27-30 2011, p 146-152

27. Mollov S, Boom T, Cuesta F, Ollero A, Babuska R (2002) Robust stability constraints for fuzzy model predictive control. IEEE Trans Fuzzy Syst 10:50-64

28. Tanaka K, Ikeda T (1998) Absolute stability conditions in a fuzzy phase-lead compensation and their extension to MIMO systems. IEEE Trans Ind Electron 45:333-340

29. Andúar JM, Bravo IM, Peregrín A (2004) Stability analysis and synthesis of multivariable fuzzy systems using interval arithmetic. Fuzzy Sets Syst 148:337-353

30. Sugeno M (1999) On stability of fuzzy systems expressed by fuzzyrules with singleton consequents. IEEE Trans Fuzzy Syst 7:201-224

31. Feng G (2006) A survey on analysis and design of model-based fuzzy control systems. IEEE Trans Fuzzy Syst 14:676-697

32. Fuh CC, Tung PC (1997) Robust stability analysis of fuzzy control systems. Fuzzy Sets Syst 88:289-298

33. Khalil HR (1992) Nonlinear systems. Macmillan, New York

34. Ban X, Gao XZ, Huang X (2007) Vasilakos AV stability analysis of the simplest Takagi-Sugeno fuzzy control system using circle criterion. Inf Sci 177:4387-4409

35. Driankov D, Hellendoor H, Reinfrank M (1993) An introduction to fuzzy control. Springer, Berlin

36. Espada A, Barreiro A (1999) Robust stability of fuzzy control systems based on conicity conditions. Automatica 35:643-654

37. Aracil J, Gordillo F (2000) Stability issues in fuzzy control. Physica, Heidelberg

38. Tanaka K, Sugeno M (1992) Stability analysis and design of fuzzy control system. Fuzzy Sets Syst 45:135-156

39. Tong S, Li HH (2002) Observer-based robust fuzzy control of nonlinear system with parametric uncertainties. Fuzzy Sets Syst 131:165-184

40. Ahn CK (2012) Exponential $\mathrm{H} \infty$ stable learning method for Takagi-Sugeno fuzzy delayed neural networks: a convex optimization approach. Comput Math Appl 63:887-895

41. Maeda M, Murakami S (1991) Stability analysis of fuzzy control systems using phase planes. Jpn J Fuzzy Theory Syst 3: 149-160

42. Lin HR, Wang WJ (1998) L2-stability analysis of fuzzy control systems. Fuzzy Sets Syst 100:159-172

43. Li Y, Yonezawa Y (1991) Stability analysis of a fuzzy control system by the hyperstability theorem. Jpn J Fuzzy Theory Syst 3:209214

44. Popov VM (1973) Hyperstability of control systems. Springer, Berlin

45. Calcev G, Gorez R, De Neyer M (1998) Passivity approach to fuzzy control systems. Automatica 34:339-344

46. Wang HO, Tanaka K, GriGn M (1996) An approach to fuzzy control of nonlinear system: stability and design issues. IEEE Trans Fuzzy Syst 4:14-23 
47. Huang CP (2005) Stability analysis of discrete singular fuzzy systems. Fuzzy Sets Syst 151:155-165

48. Liu H, Sun F, Sun Z (2005) Stability analysis and synthesis of fuzzy singularly perturbed systems. IEEE Trans Fuzzy Syst 13:273-284

49. Dong J, Yang G (2009) H $\infty$ control design for fuzzy discrete-time singularly perturbed systems via slow state variables feedback: an LMI-based approach. Inf Sci 179:3041-3058

50. Xu S, Lam J (2004) Robust stability and stabilization of discrete singular systems: an equivalent characterization. IEEE Trans Autom Control 49:568-574

51. Xu S, Song B, Lu J, Lam J (2007) Robust stability of uncertain discrete-time singular fuzzy systems. Fuzzy Sets Syst 158:23062316

52. Chen J, Sun F, Yin Y, Hu C (2011) State feedback robust stabilisation for discrete-time fuzzy singularly perturbed systems with parameter uncertainty. IET Control Theory Appl 5:1195-1202

53. Li THS, Lin KJ (2004) Stabilization of singularly perturbed fuzzy systems. IEEE Trans Fuzzy Syst 12:579-595

54. Yang GH, Dong J (2008) Control synthesis of singularly perturbed fuzzy systems. IEEE Trans Fuzzy Syst 16:615-629

55. Siettos CI, Bafas GV (2002) Semiglobal stabilization of nonlinear systems using fuzzy control and singular perturbation methods. Fuzzy Sets Syst 129:275-294

56. Benrejeb M (2010) Stability study of two level hierarchical nonlinear systems, plenary lecture 12th IFAC Symposium on Large Scale Systems : Theory And Applications. Lille, July 2010

57. Liu VT, Lin CL (1994) Robust stabilization for composite observerbased control of discrete systems. Automatica 30:877-881

58. Kando H, Iwazumi T (1986) Multirate digital control design of an optimal regulator via singular perturbation theory. Int J Control 44:1555-1578

59. Li THS, Li JH (1992) Stabilization bound of discrete two-timescale systems. Syst Control Lett 18:479-489

60. Litkouhi B, Khalil HK (1984) Infinite-time regulators for singularly perturbed difference equations. Int J Control 39:587-598

61. Chow J, Kokotovic P (1976) A decomposition of near-optimum regulators for systems with slow and fast modes. IEEE Trans Autom Control 21:701-705

62. Mahmoud MS (1982) Design of observer-based controllers for a class of discrete systems. Automatica 18:323-328

63. Kim E, Kim D (2001) Stability analysis and synthesis for an affine fuzzy system via LMI and ILMI: Discrete case, IEEE Transactions on Systems, Man, and Cybernetics. Part B Cybern 31:132-140

64. Feng G (2004) Stability analysis of discrete time fuzzy dynamic systems based on piecewise Lyapunov functions. IEEE Trans Fuzzy Syst 12:22-28

65. Zhou SS, Feng G, Lam J, Xu SY (2005) Robust H-infinity control for discrete fuzzy systems via basis-dependent Lyapunov functions. Inf Sci 174:197-217

66. Guerra TM, Vermeiren L (2004) LMI-based relaxed nonquadratic stabilization conditions for nonlinear systems in the TakagiSugeno's form. Automatica 40:823-829

67. Johansson M, Rantzer A, Arzen KE (1999) Piecewise quadratic stability of fuzzy systems. IEEE Trans Fuzzy Syst 7:713-722

68. Dieulot JY, El Kamel A (2002) Borne P study of the stability of fuzzy controllers by an estimation of the attraction regions: a vector norm approach. Math Probl Eng 8:221-231
69. Borne P, Richard JP, Radhy NE (1996) Stability, stabilization, regulation using vector norms, nonlinear systems, stability and stabilization. Chapman and Hall, Boca Raton

70. Perruquetti W, Richard JP, Grujic LJT, Borne P (1995) On practical stability with the settling time via vector norms. Int J Control 62:173-189

71. Grujic LJT, Gentina JC, Borne P (1976) General aggregation of large-scale systems by-vectorLyapunov functions and vector norms. Int J Control 24:529-550

72. Borne P, Richard JP (1990) Local and global stability of attractors by use of the vector norms, The Lyapunov Functions Method and Applications. J.C. BALTZER, A.G. Sc. Rub. Co. 8:53-62

73. Borne P, Benrejeb M (2008) On the representation and the stability study of large scale systems. Int J Comput Commun Control 3(Suppl. Issue - ICCCC 2008):55-66

74. Borne P (1987) Non-linear systems stability : vector norm approach, systems and control encyclopedia. Pergamon, Oxford

75. Grujic LT, Richard JP, Borne P, Gentina JC (2004) Stability domains. Chapman and Hall, London

76. Benrejeb M, Borne P (1978) On an algebraic stability criterion for non-linear processes. Interpretation in the frequency domain, Proceedings of the Measurement and Control International Symposium MECO'78, Athens, 26-29 June 1978, pp 678-682

77. Benrejeb M, Gasmi M (2001) On the use of an arrow form matrix for modelling and stability analysis of singularly perturbed nonlinear systems. Syst Anal Model Simul 40:509-525

78. Benrejeb M, Gasmi M, Borne P (2005) New stability conditions for TS fuzzy continuous nonlinear models. Nonlinear Dyn Syst Theory 5:369-379

79. Benrejeb M, Soudani D, Sakly A, Borne P (2006) New discrete Tanaka Sugeno Kang fuzzy systems characterization and stability domain. Int J Comput Commun Control I:9-19

80. Borne P, Vanheeghe P, Duflos E (2007) Automatisation des processus dans l'espace d'état. Editions Technip, Paris

81. Filali RL, Hammami S, Benrejeb M, Borne P (2012) On synchronization, anti-synchronization and hybrid synchronization of $3 \mathrm{D}$ discrete generalized $\mathrm{H} \frac{1}{2}$ non map. Nonlinear Dyn Syst Theory 12:81-96

82. Gil MI (2000) On Aizerman-Myshkis problem for systems with delay. Automatica 36:1669-1673

83. Gil MI, Medinab R (2002) Aizerman's problem for discrete systems. Appl Anal Int J 81:1367-1375

84. Robert F (1966) Recherche d'une M-matrice parmi les minorantes d'un opérateur liéaire. Numerische Mathematik 9:188-199

85. Robert F (1964) Normes vectorielles de vecteurs et de matrices. Revue Française de Traitement de l'Information Chiffres 7: 261-299

86. Gentina JC, Borne P, Laurent F (1972) Stabilité des systémes continus non linéaires de grande dimension. RAIRO Revue jaune J3: 69-77

87. Kotelyanski DM (1952) Some properties of matrices with positive elements. Matematicheskii Sbornik 31:497-505 [in Russian] 